\title{
ON THE FORCE AND MOMENT ACTING ON A BODY IN SHEAR FLOW*
}

\author{
By YUNG-HUAI KUO (California Institute of Technology)
}

Recently, H. S. Tsien solved the problem ${ }^{1}$ of a Joukowsky airfoil in a steady, twodimensional flow of constant vorticity distribution. It is interesting to note that the hydrodynamical forces can be expressed in a form similar to the well known Blasius' theorem, involving contour integration of the complex potential function. The following derivation of the formulae is believed to be simpler than that of Tsien.

1. Equations of motion. Let $u$ and $v$ be the velocity components parallel to the $x$-and $y$-axis, respectively. In the case of two-dimensional steady motion, the Eulerian dynamical equations are:

$$
\begin{aligned}
& u \frac{\partial u}{\partial x}+v \frac{\partial v}{\partial x}-v\left(\frac{\partial v}{\partial x}-\frac{\partial u}{\partial y}\right)=-\frac{1}{\rho} \frac{\partial p}{\partial x} \\
& u \frac{\partial u}{\partial y}+v \frac{\partial v}{\partial y}+u\left(\frac{\partial v}{\partial x}-\frac{\partial u}{\partial y}\right)=-\frac{1}{\rho} \frac{\partial p}{\partial y}
\end{aligned}
$$

where $p$ is the pressure and $\rho$, the density of the fluid. The equation of continuity is

$$
\frac{\partial u}{\partial x}+\frac{\partial v}{\partial y}=0
$$

For the type of shear flow considered by Tsien, ${ }^{1}$ the vorticity is constant everywhere in the field and equal to $-k$. Thus

$$
\frac{\partial v}{\partial x}-\frac{\partial u}{\partial y}=-k, \quad k>0
$$

At the first sight, it seems that the problem might not be definite as ane has four equations for three variables. By eliminating $p$ between Eqs. (1.1) and (1.2), however, the result can be reduced to Eq. (1.3) by means of Eq. (1.4). This shows that any solution which satisfies Eqs. (1.3) and (1.4) is consistent with Eqs. (1.1) and (1.2).

To simplify the problem, the solution is written in the following form:

$$
\begin{aligned}
u & =k y+u^{\prime}, \\
v & =v^{\prime} .
\end{aligned}
$$

Then Eqs. (1.3) and (1.4) reduce to

$$
\begin{aligned}
& \frac{\partial u^{\prime}}{\partial x}+\frac{\partial v^{\prime}}{\partial y}=0 \\
& \frac{\partial v^{\prime}}{\partial x}-\frac{\partial u^{\prime}}{\partial y}=0 .
\end{aligned}
$$

* Received June 21, 1943.

${ }^{1}$ H. S. Tsien, Symmetrical Joukowski airfoils in shear flow, Quarterly Appl. Math., 1, 129 (1943). 
These equations are satisfied by

or

$$
u^{\prime}=\frac{\partial \psi}{\partial y}, \quad v^{\prime}=-\frac{\partial \psi}{\partial x}
$$

$$
u^{\prime}=\frac{\partial \varphi}{\partial x}, \quad v^{\prime}=\frac{\partial \varphi}{\partial y} ;
$$

where $\psi$ and $\varphi$ are the imaginary and real parts of the complex potential $F(z)$; namely,

$$
\varphi+i \psi=F(z), \quad z=x+i y
$$

and

$$
u^{\prime}-i v^{\prime}=w^{\prime}(z) .
$$

For a given problem the function $F(z)$ is so determined that the velocity component normal to the contour of the body is zero.

By virtue of Eqs. (1.4), (1.5), and (1.6), Eqs. (1.1) and (1.2) give

$$
p=-\frac{\rho}{2} q^{\prime 2}-\rho k u^{\prime} y+\rho k \psi,
$$

where $q^{\prime 2}=u^{\prime 2}+v^{\prime 2}$, and the constant of integration is absorbed in $\psi$.

2. Force and moment. If the motion is two-dimensional and steady, the components of the hydrodynamical force and moment ${ }^{2}$ acting on the body are given by

$$
\begin{aligned}
X & =-\oint p d y-\rho \oint u(u d y-v d x), \\
Y & =\oint p d x+\rho \oint v(v d x-u d y), \\
M & =\oint p(x d x+y d y)-\rho \oint\left(-v^{2} x d x-u^{2} y d y+u v y d x+u v x d y\right),
\end{aligned}
$$

where the contour integrals are taken along a closed curve containing the body. Using Eqs. (1.5), (1.6) and (1.13), the above equations can be written as:

$$
\begin{aligned}
X= & -\frac{\rho}{2} \oint\left[\left(u^{\prime 2}-v^{\prime 2}\right) d y-2 u^{\prime} v^{\prime} d x\right]-\rho k \oint\left[\left(\psi+u^{\prime} y\right) d y-v^{\prime} y d x\right], \\
Y= & -\frac{\rho}{2} \oint\left[\left(u^{\prime 2}-v^{\prime 2}\right) d x+2 u^{\prime} v^{\prime} d y\right]+\rho k \oint\left[\left(\psi-u^{\prime} y\right) d x-v^{\prime} y d y\right], \\
M= & -\operatorname{Re}\left[\frac{\rho}{2} \oint z w^{\prime 2} d z\right] \\
& +\rho k \oint\left[\left(\psi-u^{\prime} y\right)(x d x+y d y)-\left(v^{\prime} y x-2 u^{\prime} y^{2}\right) d y+v^{\prime} y^{2} d x\right] .
\end{aligned}
$$

${ }^{2}$ W. F. Durand, Aerodynamic theory, vol. 2, Springer, Berlin, 1935, pp. 31-33. 
If only bodies with closed boundary are considered, no sources can exist within the field of flow. Then the stream function $\psi$ is single-valued, and

$$
\begin{aligned}
& \oint \psi d x=\oint x\left(v^{\prime} d x-u^{\prime} d y\right), \\
& \oint \psi d y=\oint y\left(v^{\prime} d x-u^{\prime} d y\right) .
\end{aligned}
$$

From these relations, it is not difficult to deduce

$$
\begin{aligned}
X= & -\frac{\rho}{2} \oint\left[\left(u^{\prime 2}-v^{\prime 2}\right) d y-2 u^{\prime} v^{\prime} d x\right], \\
Y= & -\frac{\rho}{2} \oint\left[\left(u^{\prime 2}-v^{\prime 2}\right) d x+2 u^{\prime} v^{\prime} d y\right] \\
& +\rho k \oint\left[v^{\prime}(x d x-y d y)-u^{\prime}(y d x+x d y)\right], \\
M= & -\operatorname{Re}\left[\frac{\rho}{2} \oint z w^{\prime 2} d z\right] \\
& +\frac{\rho k}{2} \oint\left[-u^{\prime}\left\{\left(x^{2}-y^{2}\right) d y+2 x y d x\right\}+v^{\prime}\left\{\left(x^{2}-y^{2}\right) d x-2 x y d y\right\}\right] .
\end{aligned}
$$

These at once suggest the following alternative expressions:

and

$$
X-i Y=\frac{i \rho}{2} \oint w^{\prime 2} d z+i \operatorname{Im}\left[\rho k \oint w^{\prime} z d z\right]
$$

$$
M=-\operatorname{Re}\left[\frac{\rho}{2} \oint z\left(w^{\prime}-\frac{i k z}{2}\right)^{2} d z\right] .
$$

Eqs. (2.10) and (2.11) may be regarded as an extension of Blasius' theorem. They can be easily identified with the expressions given by Tsien. ${ }^{1}$ The calculation of force and moment, however, can be simplified to a certain extent by using these new expressions.

The writer wishes to thank Dr. H. S. Tsien for the use of his paper before publication and for his helpful discussions. 